http://jmscr.igmpublication.org/home/ ISSN (e)-2347-176x ISSN (p) 2455-0450 crossref DOI: https://dx.doi.org/10.18535/jmscr/v7i8.124

\title{
Ignorance is not bliss- A neglected inguinal hernia with obstruction can lead to the eternal home
}

\author{
Author \\ Dr K.Venkata Vigneswara Rao M.S. (Gen Surgery) ${ }^{1}$, Dr M. Ramachandra DNB (Gen \\ Surgery) ${ }^{* 2}$, Dr B Raviteza MBBS (M.S.) ${ }^{3}$ \\ ${ }^{1}$ Associate Professor, Department of General Surgery, Government Medical College, RIMS, Kadapa, AP \\ ${ }^{2}$ Associate Professor, Department of General Surgery, Government Medical College, RIMS, Kadapa. AP \\ ${ }^{3}$ PG Department of General Surgery, Government Medical College, RIMS, Kadapa, AP \\ *Corresponding Author \\ Dr M. Ramachandra DNB (Gen Surgery) \\ Associate Professor, Department of General Surgery, Govt. Medical College, RIMS, Kadapa, AP, India
}

\begin{abstract}
In our country, a large part of patients still neglects inguinal hernia due to the low level of public awareness about the danger of obstruction and strangulation. Even with better pre and postoperative care, improved anesthetic management and advanced surgical techniques, the emergency repair of an obstructed hernia is associated with poor prognosis and a high rate of postoperative complications. This study is to assess various risk factors and therapeutic strategies for better outcome of the patients presented with an obstructed inguinal hernia at our setup. A total of 74 inguinal hernia patients with obstruction were evaluated. Majority of the patients were in the fifth and sixth decade. More than 50\% of cases belong to low socio-economic strata, who do heavy work. Right-sided Indirect hernias were more common than others. Chronic cough, constipation, prostatic enlargement are common causes of increased intra-abdominal tension seen in more than $60 \%$ of cases. All of the cases were operated as an emergency procedure. Viable bowel was seen $75.46 \%$ of cases. Bowel resection and end-to-end anastomosis were done in all cases of nonviable bowel. Patients presented early with the only obstruction, had an excellent prognosis; But the danger group, where symptomatology is vague and associated with strangulation had a higher rate of complications and mortality.
\end{abstract}

Keywords: Inguinal Hernia, Intestinal Obstruction,Neglected Hernia, Bowel Resection.

\section{Introduction}

In an obstructed inguinal hernia, the hernia is associated with intestinal obstruction due to occlusion of the lumen of bowel with unimpaired blood supply to the contents, and It becomes Strangulated with the arrest of blood supply to the contents. Intestinal obstruction is absent in case of omentocele, Richter's hernia, and Littre's hernia.
Obstructed Inguinal hernia is more common in the older age group with indirect, recurrent hernias and is of the closed-loop type. Smoking is a significant risk factor as it is associated with abnormal collagen metabolism. Another critical factor in the outcome of is a presence of comorbid chronic diseases like Chronic cough Constipation, Bladder neck or urethral obstruction, Severe muscular effort, 
Parturition, Vomiting, ascites as they contribute to an increase of intraabdominal pressure. The local weakness of the abdominal wall with Impaired Shutter mechanism leads to "give way" at the internal inguinal ring when intra-abdominal pressure rises and intra-abdominal contents, which get stuck up, got obstructed. Presence of preformed Congenital Sac (an unobliterated portion of processus vaginalis) primarily attributed in younger age patients where occupational strain, increased intrabdominal tension are not present. Late hospitalization is generally considered as an essential factor for determining the level of intestinal resection and subsequent morbidity and mortality. A higher rate of morbidity and mortality is seen in patients with higher ASA grade.

An essential aspect in the diagnosis of obstructed inguinal hernia is to differentiate it from the strangulated inguinal hernia. Irreducibility may persist for years or decades without great inconvenience as a result of adhesions developing between the contents and the sac. Adhesions between the contents of the hernia and the peritoneal lining of the sac can provide a tethering point that entraps the hernia contents and predisposes to intestinal obstruction and strangulation. Strangulation leads to gangrene and, perforation of the bowel and is an indication for urgent repair.

A plain supine abdominal radiograph may give a clue to the diagnosis with a clear cut-off visible in the bowel. Bedside ultrasound showed bowel loops within the hernia sac. Ultrasound features suggestive of bowel strangulation are thickening of the hernial sac, fluid within the sac, edematous thick bowel walls, loss of peristalsis A computed tomography (CT) with oral, intravenous and enema contrast is more sensitive than a plain radiograph in diagnosing the obstruction, and may also shed light on the aetiology as well. Preoperative laboratory investigations should include full blood count to assess leucocytosis as an indicator of intestinal infarction and haematocrit to assess hydration. Blood biochemistry may reveal features suggestive of dehydration, such as electrolyte imbalance or raised creatinine and urea.

Treatment begins with resuscitation and this period must be finely judged and is essential to bring deranged parameters to be normal to near normal. The merits of optimising the patient's state of hydration, electrolyte balance, and cardiopulmonary status must be balanced against the systemic toxic complications of unresected, infarcted bowel.

Open tissue repair is commonly utilised for early obstructed inguinal hernias with viable contents however, other surgical treatment modalities are becoming more widely accepted including the use of prosthetic mesh The difference in complications and recurrence following repair of acutely incarcerated inguinal hernias using these techniques is an area of ongoing research and debate in the general surgery community, with a wide variation in practice patterns.

Non viability of contents will necessitate conversion into a laparotomy followed by the release of the constricting hernia ring, reduction of the contents of the sac, resection, and reanastomosis. Precautions should be taken to avoid contamination of the general peritoneal cavity by gangrenous bowel or intestinal contents. In the majority of cases, circulation to the intestine is re-established with the release of constriction, and viability returns. If viability is doubtful, resection is performed

\section{Methods}

This study is a retrospective observational study, conducted in the Department of General Surgery, Government Medical College and Hospital, RIMS Kadapa from July 2017 to June 2019. All Patients attended causality with a presentation of obstructed inguinal hernia with or without strangulation, are included in the study. Patients admitted with an irreducible hernia that got reduced spontaneously and by taxis are excluded from the study.

Patients age, sex, occupation, chief complaints with duration are taken. Mode of onset, time of arrival after symptoms, symptoms of intestinal obstruction, precipitating factors of obstruction are noted. A thorough physical examination focusing on the 


\section{JMSCR Vol||07||Issue||08||Page 734-738||August}

duration of hernia and the duration of the irreducibility of hernia, strangulation and peritonitis is recorded. All Lab reports, radiological investigations are drawn. Patients were resuscitated and subjected to different surgeries, depending on the viability of the contents. Postoperative complications are observed, and the following conclusions are made.

\section{Results and Analysis}

Our study comprises of 74 patients who have attended casualty with the presentation of Obstructed inguinal hernia with or without strangulation. Out of 74 cases, no case of a female is present with obstruction probably because of anatomical differences between the genders and their susceptibility to various types of hernia differs. The right-sided hernia is involved in 58 cases whereas 16 are on Left side. It is understandable as the testis on the right side descends later than the left and the consequently delayed closure of the processus vaginalis on the right side. Obstructed Inguinal hernia is associated with Indirect sac in 63 cases and direct type In 11 cases. More than $50 \%$ of cases belong to low socio-economic strata, who do heavy work. Chronic cough, constipation, prostatic enlargement are common causes of increased intraabdominal tension seen 41 patients, which predisposes to obstruction. Occupational stress (36 cases), Local weakness (17) and presence of congenital sac (11) are other risk variables Observed in development Obstructed inguinal hernia.

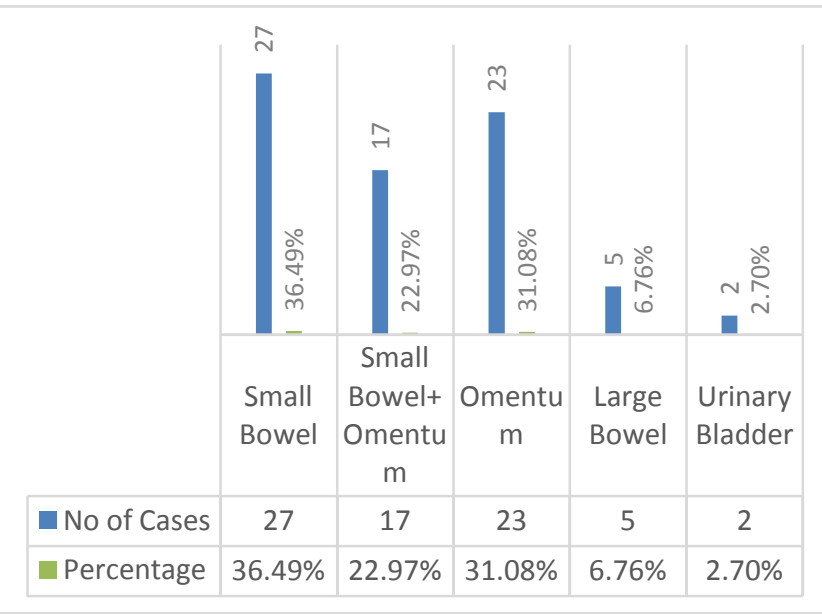

Time Interval between Onset Obstruction and Pt Seeking Treatment

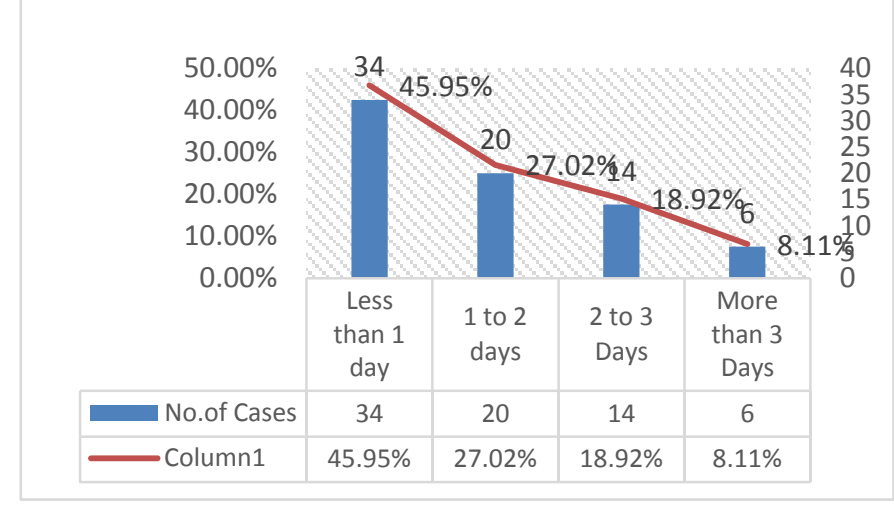

Risk variables In Obstructed Inguinal hernia

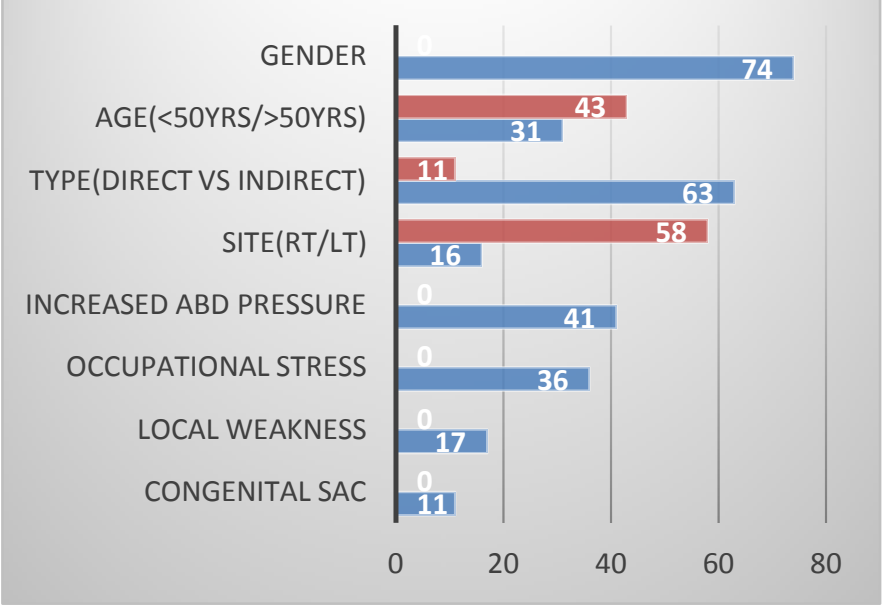

Most Patients had a duration ranging from 3 months to 10 years of swelling in the groin before developing obstruction. In one-third of cases, there is a history of reducibility of swelling on lying down or taxis by the patient himself, which have got obstructed suddenly or gradually. In $60 \%$ of cases, the obstruction is sudden. Pain and swelling are present in all most all the cases. Pain ranges from dragging, intermittent colicky to constant aching pain at the region of swelling. The duration of obstruction before patient seeking treatment usually ranges from 6 to 48 hours. Moreover, nearly 28 per cent of cases presented after 48 hours of obstruction and this danger group requires sensible decisions to stop the vicious cycle of strangulation, before going for the repair of the hernia defect.

All the cases are taken for surgery after resuscitation within 2-6 hours of admission. During surgery Site of Obstruction was noted, and the contents are examined for viability. The obstruction is at internal 
inguinal rings in 63 cases. In 7 cases obstruction is at superficial Ring remaining at in Inguinal canal. Obstructed Sac mainly contains small bowel in 27 cases, Omentam in 27, but both were present in 33 cases. Large bowel (5 cases) and Urinary bladder ( 2 cases) are also noted.

\section{Contents noted on Operative Table}

In $75.46 \%$ of cases, the contents are viable, and in these cases, contents are replaced after releasing adhesions, and hernioplasty was performed. In remaining cases, where the contents of the sac are nonviable, Omentectomy and Resection anastomosis of bowel was done along with herniorrhaphy. In the present series, hernioplasty was done in 54 cases whereas herniorrhaphy along with omentectomy done in 12 cases and herniorrhaphy along with resection and anastomosis done in 8 cases.

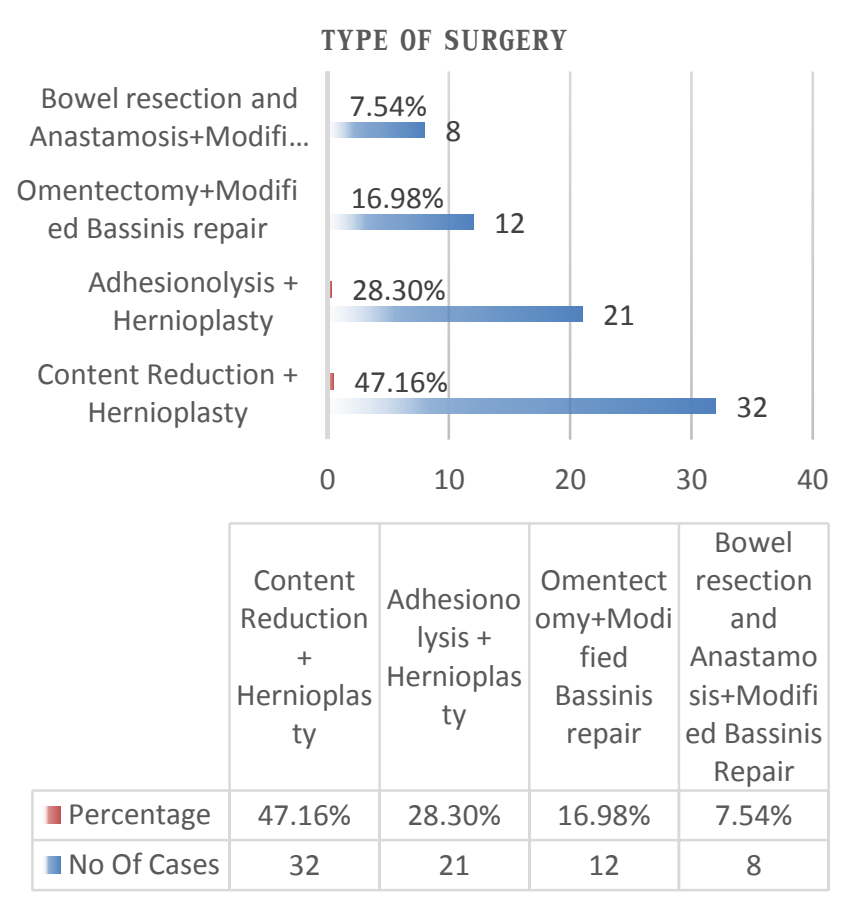

The following postoperative complications are noted. Postoperative seroma/hematoma is present in 12 patients which subsided with rest and antibiotics. Postoperative infection present in 9cases subsided with antibiotics. Respiratory disturbances comprised of non-expectorant cough; dyspnoea was observed in 6 cases. These patients were given chest physiotherapy with Antibiotics, steam inhalation nebulisation and cough suppressants. Paralytic ileus found in 2 cases due to dyselectrolemia. Paresthesias are observed in 5 cases which were managed with analgesics. The death occurred in 2 cases. Postoperative complications are commonly seen in patients with the coexisting disease, hernias of longer duration, as well as higher ASA class.

\begin{tabular}{|l|c|c|}
\hline $\begin{array}{l}\text { Post-Operative } \\
\text { Complication }\end{array}$ & $\begin{array}{c}\text { Mesh } \\
\text { repair }\end{array}$ & $\begin{array}{c}\text { Modified } \\
\text { Bassinis } \\
\text { repair }\end{array}$ \\
\hline $\begin{array}{l}\text { Post-Operative } \\
\text { Seroma/Hematoma }\end{array}$ & 5 & 7 \\
\hline $\begin{array}{l}\text { Post-Operative } \\
\text { Wound infection }\end{array}$ & 4 & 5 \\
\hline Respiratory disturbances & 3 & 3 \\
\hline Paresthesias & 2 & 3 \\
\hline Paralytic ileus & 0 & 2 \\
\hline Death & 0 & 2 \\
\hline Total & 14 & 22 \\
\hline
\end{tabular}

\section{Conclusion}

In conclusion, our data suggest that acutely obstructed or strangulated groin hernia in the elderly is a serious problem because of the high morbidity and mortality rate. Quick diagnosis and early surgical Interventions are desirable in order to avoid the complications that occur in emergency operations. All Patients with Inguinal hernia be warned and educated about the potential danger of obstruction and strangulation; this is particularly more important in elderly patients and Patients with comorbid risk factors.

\section{References}

1. Mokete $\mathbf{M}$ and Earnshaw $\mathbf{J} \mathbf{J}$. evolution of inguinal hernia surgery practicespost grad medical journal 2001;77:189-190

2. Principle'sofSurgerySchwartz's $10^{\text {th }}$ edition, 2 015, cha37:1495

3. Management of Abdominal Hernias 4e, Morten Bay-Nielsen, Andrew N. Kingsnorth, Karl A. LeBlanc. Springer Science Business Media London 201309.Andrews MJ.

4. Presentation and outcome of strangulated external hernia in a district general hospital. Br J Surg. 1981;68(3):29-32.

5. Hariprasad S, Srinivas T. Clinical study on complicated presentations of groin hernias. Int J Res Med Sci 2017;5:3303-808. 
6. Alvarez JA, Baldonedo RF, Bear IG, Sol'1s JA, Alvarez P, Jorge JI. Incarcerated groin hernias in adults: presentation and outcome. Hernia 2004;8(2):121-126. 